\title{
Remarks on neurocybernetics and its links to computing science. To the memory of Prof. Luigi M. Ricciardi
}

\author{
Roberto Moreno-Díaz ${ }^{a}$, Arminda Moreno-Díaz ${ }^{\text {,** }}$ \\ a Instituto Universitario de Ciencias y Tecnologías Cibernéticas, Parque Científico-Tecnológico, Universidad de Las Palmas de Gran Canaria, 35017 Las Palmas de G.C., Spain \\ b School of Computer Science, Campus de Montegancedo S/N, Technical University of Madrid (UPM), 28660 Madrid, Spain
}

\section{A R T I C L E I N F O}

\section{Article history:}

Received 10 October 2012

Accepted 29 November 2012

\section{MSC:}

$68 \mathrm{~T} 01$

$68 \mathrm{~T} 30$

Keywords:

Neurocybernetics

Computing science

Brain processes

\begin{abstract}
A B S T R A C T
This paper explores the origins and content of neurocybernetics and its links to artificial intelligence, computer science and knowledge engineering. Starting with three remarkable pieces of work, we center attention on a number of events that initiated and developed basic topics that are still nowadays a matter of research and inquire, from goal directed activity theories to circular causality and to reverberations and learning. Within this context, we pay tribute to the memory of Prof. Ricciardi documenting the importance of his contributions in the mathematics of brain, neural nets and neurophysiological models, computational simulations and techniques.
\end{abstract}

(C) 2013 Elsevier Ireland Ltd. All rights reserved.

\section{Introduction}

This paper explores the evolution and content of neurocybernetics, its relation to artificial intelligence, computer science and knowledge engineering. There are three contributions to the origin of cybernetics as such and the three appeared in 1943: first, Roseblueth et al.'s (1943); second, Kenneth Craik's (1943) and third McCulloch and Pitt's (1943). They established, through a kind of synergetic process, the concepts and ideas that were to mature during the Macy's Foundation Meetings and the crucial contributions by the historical participants (McCulloch, 1989; Pias, 2003). These meetings were first named "Conferences on Circular, Causal and Feedback Mechanisms in Biological Systems" and later "Conferences on Cybernetics". Macy's papers initiated and developed basic topics that are still nowadays a matter of research and inquire, ranging from goal directed activity theories to circular causality and to reverberations and learning.

From them, the many crucial contributions up to the Seventies, were from Wiener, McCulloch and his group, McKay, Von Foerster, Gordon Pask, Caianiello and many others (McCarthy et al., 1955; McCulloch et al., 1969; Moreno-Díaz and Moreno-Díaz, 2007). Minsky had established the artificial intelligence Laboratory at M.I.T., connecting the ideas of the 1953 Dartmouth Meeting and those of the neurophysiologists close to McCulloch. On the other hand,

\footnotetext{
* Corresponding author. Tel.: +34 91336 7438; fax: +34 913524819

E-mail address: amoreno@fi.upm.es (A. Moreno-Díaz).
}

Caianiello triggered the research in the mathematics of brain and neural nets. His meetings in Varenna in 1958 (Ricciardi, 1994), brought together the top of leading multidisciplinary scientists in the spirit of the original Macy's, from Wiener on. Caianiello and his disciples (Ricciardi, Marinaro, de Luca and others) produced a considerably body of ideas and formal tools to brain theory and neural nets, having the rigorous flavour of physiscs and mathematics of their original neuronic and mnemonic equations (Marinaro et al., 1995). This effort was extended by Ricciardi and disciples mainly in the estabishment and analysis of neurophysiological models based on stochastic processes, connecting them to computational simulations and to more general computational techniques. These are reflected, for example, in Ricciardi and Lánský (2003) and in many other prior and posterior of Ricciardi's rich production (see, for example, Springer EUROCAST selected papers books since 1993).

The evolution of the computing sciences, intermingled with theoretical and practical neurophysiology in the direction of bioinspired artificial intelligence, led nowadays to what is known as computational intelligence (Moreno-Díaz and Mira, 1996; MiraMira and Delgado, 2006). Here, again as in the old times, the central questions are to find the appropriate formal tools to define and clarify the understanding, formalization and mechanization of thought processes at different levels. That is, to make them somehow computable or, in McCulloch (1974) words "to find the embodiments of mind".

It is apparent that there is a large source of inspiration for problem statement and formulation in the links of neuroscience and artificial intelligence (A.I.), this one understood as a 


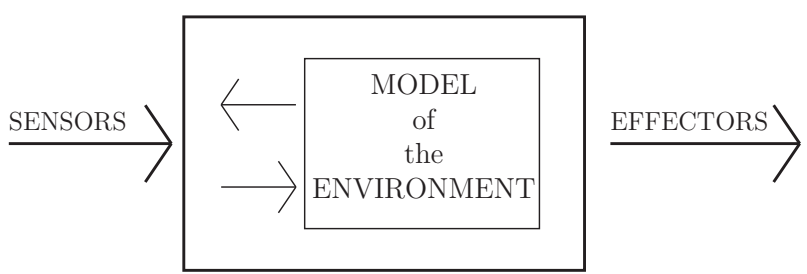

Fig. 1. Basis of symbolic A.I. agents and robotics.

simplest mechanization of thought processes. In this way, there are three paradigms of A.I. that are relevant to Neurocybernetics: the Symbolic Agent Paradigm, the Intelligent Agent Paradigm and, more clasically, the Connectivistic Approach. These and their implications are explored here. Following one of Prof. Ricciardi's main general ideas during his maturity lectures (Ricciardi, 2010), we want to emphasize the need to return in a systematic way to the original writings of the many authors, where many of the basic ideas can be found, which are still pending to be developed and which, furthermore, are a healthy source of inspiration.

This paper is dedicated to Prof. Luigi Ricciardi's Memory, to his lively, strong and serious contributions to linking mathematics and biosciences and to his valuable hospitality and friendship. We also present an acknowledgment of his many contributions to the various scientific meetings on cybernetics organized mostly in Las Palmas de Gran Canaria.

\section{The original components of cybernetics}

Neurocybernetics took off in the Forties although many of the basic ideas had been being managed in philosophic and scientific circles since the times of the Ancient Greeks. From 1943 to 1945, a kind of synergetic process was started up, triggered as the result of three basic pieces of work: first, Norbert Wiener, Arthur Rosemblueth and Julian Bigelow's study in 1943 (Rosenblueth et al., 1943), on the nature of teleological processes, where the crucial idea was that the relevant issue in a homeostatic process is the information return and not the energy return via the feedback loops.

Second, it came the work of the young British philosopher Kenneth Craik, published in the form of a small book called On the Nature of Explanation in 1943 (Craick, 1943). He offered a pursuit of a Theory of Knowledge which would be contrastable like any other Natural Science. He was not completely successful in achieving this aim but he did, however, establish the rational bases upon which all the theories and models of systems of artificial behaviour have since then been built. Craik offered a clear and powerful framework within which to express the acquisition, processing, storage, communication and use of knowledge. For a symplified diagram of Craik's constructivistic model (see Fig. 1).

And third, the work of Warren McCulloch and Walter Pitts, A Logical Calculus of the Ideas Immanent in Nervous Activity, which was also published in 1943 (McCulloch and Pitts, 1943). They elaborated the concept of a "formal neuron" introducing the use of mathematical logic to explain neural mechanisms. The "formal neuron" response is, in fact, equivalent to a symbolic proposal with respect to the corresponding stimulus and which allows for a neural network to be considered as a logical system capable of handling symbols and elevating them to the level of the logic required for proposals (see Fig. 2). For further discussion on this seminal paper see, e.g., Piccinini (2004), Schlatter and Aizawa (2007).

The first of the three works mentioned earlier is the crucial paper for the emergence of cybernetics. It was presented at the first Macy Foundation meeting in New York City and it was published the following year by Rosenblueth et al. (1943).

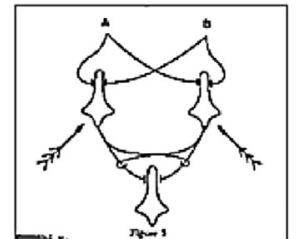

The formal Neuron is a logical unit that embodies the basic properties of excitation, inhibition, threshold and "all or one" inputs and outputs

A neural net with loops it is equivalent to what later will be called a "finite automaton" with a modular realization with the simplest units.
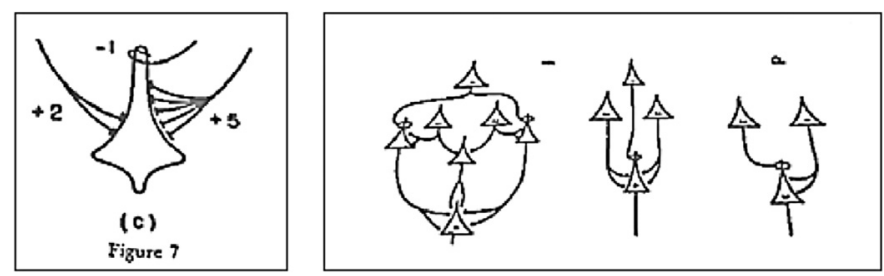

Fig. 2. Origins of connectivism.

Rosemblueth and McCulloch had reached with the Josiah Macy Foundation an important agreement to organize a yearly interdisciplinary meeting. Before they started, there was late in 1942 a meeting of engineers, physiologists and mathematicians at Princeton, referred by Wiener in the Introduction of his book of 1949 , Cybernetics (Wiener, 1948). There, McCulloch says, he met John von Neumann.

The Macy Foundation Conferences started under the name "Conferences on Circular, Causal and Feedback Mechanisms in Biological and Social Systems", which was changed to "Conferences on Cybernetics" in 1949. These series of stimulating and constructive Conferences run until 1953. They established a new conception for treating living and non-living machines, which with more or less successes, failures and redefinitions, came to our days. It would be fruitful to dig into that remarkable source of ideas and inspiration, but only some of the Transactions are available. As Von Foerster said "The Conferences have became an oral tradition, a myth". Today, the consequences of that myth can be found in McCulloch and other attendants' essays. Many of the attendants to the Conferences may be considered the real foundations of Cybernetics. Some names are: W. Ross Ashby, Y. Bar-Hillel, Julian Bigelow, Jan Droogleever-Fortuyn, W. Grey Walter, Rafael Lorente de Nó, Donald MacKay, Warren McCulloch (Chairman of the Conferences), J.M. Nielsen, F.S.C. Northrop, Linus Pauling, Antoine Remond, Arturo Rosemblueth, Claude Shannon, Heinz Von Foerster, John Von Neumann, Norbert Wiener. From that time, McCulloch was advisor and friend of the operational research pioneer Stafford Beer.

From the Macy's Conferences on, there were a number of crucial subjects and problems, raised and discussed in the many sessions. Among them, there were the concepts of regulation, homeostasis and goal directed activity, the transmission of signals and communication in machines and nervous systems or the raise of Neural Nets and Automata Theory. Regarding to nervous systems organization, the ideas of reverberating and closing loops to explain brain activity were established there. These ideas generated concepts and theories on circular causality in Economics and in the polling of public opinion.

The analysis of conflict between motives in psychiatry led to the developing of concepts like heterarchy of values in mental processes. Also, the ideas of content addressable memory, active or reverberating memories and the consideration of learning as changes in transition probabilities among states, were inspired from Biology to became terms applicable to machines. In sum, there were a considerable and rich flow of new ideas and concepts to be applied both to machine and to living systems.

Neurocybernetics evolved with powerful input from the Theory of Communication of Shannon and key figures in the field of Computer Science such as Von Neumann and his application to 
questions of computability, performability, capacity for reproduction and reliability of functioning (Von Neumann, 1956). McCulloch and Von Neumann were personal friends and the former delighted a great deal in recounting the anecdote of how they began their work together on reliability of functioning, probabilistic logic and probabilistic computing.

In the Fifties, McCulloch held the chair of Psychiatry at the University of Illinois at Chicago. One night, he, Von Neumann and some colleagues were not in perfect shape after a copious dinner. McCulloch suddenly stopped the conversation dead and commented something on its effect: "The thresholds of neurones are now very, very low. Nevertheless, neurons are still computing reasonably reliable. What can there be in the brain, in its modular structure and links, which makes it such a reliable piece of machinery in spite of failure in threshold levels and components?"

A magnificent piece of work called Agathe Tyche: The lucky reckoners (McCulloch, 1959), offers a fair overview of much of his philosophy with respect to ways of building reliable machinery from unsafe components. The classic by Cowan called Reliable Computation With Unreliable Elements (Cowan, 1966), and almost all of his later work on reliable computing was the result of McCulloch's expansion of Von Neumann's original concept.

Master contributions in the 50s and 60s include, among others, those by Ross Ashby, Heinz von Foerster and Eduardo Caianiello. Ashby's concept of homeostatic machines is fundamental for the development of mathematical Cybernetics, as well as the ideas developed in his classical book "Design for a Brain" (Ashby, 1952). Von Foerster was a physicist who became cybernetician after serving as secretary to the Macy's Foundation Conferences and editor of the Transactions. His contributions on second order cybernetics or cybernetics of observing systems are crucial to understand complex non trivial machines and systems (Von Foerster, 1974). Third, Eduardo Caianiello's neuronic and mnemonic equations for neural dynamics and for learning (Caianiello, 1961).

Around 1965, the office of McCulloch in the Electronic Research lab at the MIT was a kind of breathtaking classroom both for the quality of the science produced and for the incredible people who filed through it. All of the greats of Cybernetics were there: Colin Cherry, Donald McKay, Patrick Meredith, Von Foerster, Gordon Pask, Eduardo Caianiello, to name only a few. Marvin Minsky and Seymour Papert set up the MAC project in artificial intelligence in a nearby lab (Minsky and Papert, 1969).

After the problems on the reliability of functioning, which reached some acceptable solutions, the theory of neural networks faced up to the question of dynamic memory. This problem refers to oscillations in networks, expressly constructed to provoke controlled oscillations, which serve as a support to the dynamic storage of information. The initial logical problem was to find the maximum theoretical number of ways of oscillation in a non-linear, discrete and arbitrary network of $N$ formal neurons. Schnabel found it was a number which grows extraordinarily when the number of formal neurons is increased (Schnabel, 1965). For example, for two neurons, there are twenty oscillation modes: for three, there are 6.024 modes, i.e., three neurons could "store" 6.024 different models, each of which could be evoked by different external inputs. We say, "it could" because we still have to show that a network of fixed anatomy could be designed in a way that incorporates all the modes of oscillation. This was proved in 1966 via the theorem of synthesis, using formal neurons with afferent interaction introduced by Blum (1961).

By the year 1969, the theory of formal Neural Networks was considered, from the logical perspective, to be a closed matter mainly due to the introduction of the so-called functional matrices (Moreno-Díaz and McCulloch, 1969). They allowed, transparently, the demonstration of equivalence between determinist and probabilistic robots and networks of formal neurons with feedback, via constructive theorems. There was only one formal gap and it consisted in the fact that certain probabilistic machines had no counterpart in the logical networks of formal neurons unless an additional probabilistic codifier was incorporated into the network, previous to the input to the networks if unless the "outside" world (outside the neural) had a non-deterministic nature and, what is worse, a nature which depends on the structure of the network of formal neurons. In other words, that there are probabilistic robots which cannot be duplicated in the networks of formal neurons with afferent interaction. In fact and in the practical totality of the applications, the subject is not completely relevant. But from the theoretical perspective, it is inadmissible since we could not defend that the logical model of McCulloch and Pitts was an appropriate model to represent the activity of the brain at the computational level of coding and communication.

This gap was acknowledged, but the subject was set aside due to the fact that neural networks lost scientific interest from the end of the Seventies to the mid Eighties. In 1983, a doctorate student in Maths, took up the subject again and proved that, if interaction between axons was admitted, then the theory was complete i.e., a network of formal neurons with feedback would duplicate any arbitrary robot, be it deterministic, probabilistic or non-deterministic (Moreno-Díaz and Hernández-Guarch, 1983). This effect of output interaction was added elegantly to the interaction of afferents (inputs) of Blum dating back to 1962. This ended the so called McCulloch's Program I, the Logical Program.

McCulloch's Program II is more realistic and can be considered as brain theory at the level of Systems Sciences. The prototype paper is the 1947 paper by McCulloch and Pitts entitled "How we know Universals" (Pitts and McCulloch, 1947), as well as his and Kilmer's subsequent work on modelling the reticular formation (Kilmer and McCulloch, 1964). Actually, as Norbert Wiener says in the Introduction to his book Cybernetics, McCulloch was after an apparatus to read aloud a printed page, which, because the necessary invariances, was a definite analogue to the problem of Gestalt's form perception. He designed a device that made G. von Bonin ask if it was a diagram of the fourth layer of the visual cortex. A typical neurocybernetic solution.

Program II can be simply stated as follows: from a subsystem of the nervous system, define the most precisely its functions and try to find a cooperative, reliable granular structure to perform said functions. That will be a true theoretical neural net. Program II can be formulated for the artificial as well, so that it provides for systematic reasonable ways to solve problems by means of artificial "neural nets" of computing modules.

As it is known, in the Eighties, there was an upsurge in neural computing which, we believe, was due to one basic cause: the growing availability of microcomputers at a very low cost so that hundreds and even thousands of them could be linked up in parallel processing networks each with functions much more complex than the formal neurons of McCulloch and Pitts and the addenda. Anyway, we should not forget the fact that the classic theory is complete at a logical level and by offering greater computing potential to the basic units, the maximum we arrive at is a reduction in the number of units needed for the practical working of an artificial system apart from an increase in the speed of design. The only crucial element which had not been contemplated and which was easy to incorporate, and was incorporated in the famous Perceptrons of the 60 s, was the capacity of changing synaptic weights through learning.

As it is well known, this resurgence of neural networks as systems of distributed granular computing finds application in technological fields ranging from processing and treatment of signals (voice, image), systems of artificial vision, in robots and in control. We however believe that most works on artificial neural nets are irrelevant ways of solving problems using non-optimal 
tools. It is our believe that significant progress in artificial neural net theory (or modular distributed computation) requires to proceed strictly according to McCulloch's Program II.

\section{From neurocybernetics to bioinspired artificial intelligence}

The aims of Neurocybernetics are essentially the understanding of neural behaviour at different levels, by constructing models and theories. If we add the obvious condition that these models and theories are computable, in order to embody them in a physical structure, we can conclude that these are also the aims of the so more recently called Computational Neuroscience. Thus the range in what Neurocybernetics acts goes from membrane phenomena to perceptual and cognitive, and to behavioural and social processes.

The neural function is a really complex phenomenon and its characterization requires, as a norm, meticulous approaches both at the level of tools and methods to be applied as in accepting or choosing the parameters which are considered necessary when describing and trying to explain this function. Also care should be taken when considering the scope of possible validity of conclusions reached via the theoretical and experimental approaches adopted. This is equivalent to saying that any theory with respect to the nervous system is limited a priori by the conceptual tools. To exaggerate, we should not attempt to explain the capacity for resolution of problems of the nervous system using, for example, non-linear differential-integral equations. Nor can we delve deeper into the properties of the neural membrane using the logic of relationships.

Thus, we cannot deny the historic role played of action potential registers from the Fifties since they have allowed for a physical knowledge of the carrier substratum of messages. But it is illegitimate to work from them to deduce high level properties or to try to build functional models of the brain. It would be, albeit an unfair comparison, like using statistics of the pulses which appear in a data bus or computer commands to deduce the algorithmic base of the programme solving a problem in RAM.

We can sum up this structure of Neurocybernetics levels in a way which indicates what are the appropriate tools for each level, keeping in mind that a notable change in level cannot be allowed in the theory without changing tools. But, if prudent, in the practical research into the brain and artificial machines which we wish to make work like the brain, we can skip the level slightly.

The most basic level (where computational machines still do not appear, strictly, apart from as tools) is the level of the neurotransmitters, membrane phenomena and action potentials. Tools present in this level are Biochemistry and Biophysics. Then comes Biophysics of Neural codes and multiple codes, where this is a word used in neurophysiology to indicate multiplex. Then we move onto biophysics and signal processing. We continue through sensorial codes, decoding in motor and glandular effectors and the code of advanced peripheral neurons such as the ganglion cells in the retina. We are now in the realm of signal theory almost at the level of logic. Then, we have the neural net level, the interaction of input and output of the neurons themselves, and the coordination of the output (effectors). We are now at the level of the language of logic bordering on symbolic languages and, finally, we come to the central cortex neural code, the cooperative processes between masses of brain tissue, the extraction of Universals and the social processes of interaction between neuron masses. We are at the level of Symbolic language. The structure in levels is summarized in Fig. 3. Upper square bounds the more classical formal tools of computational neuroscience. Lower square bounds techniques close to artificial intelligence tools.

\begin{tabular}{c|ll|}
\multicolumn{1}{c}{$\begin{array}{c}\text { COMPUTING } \\
\text { AS } \\
\text { TOOLS }\end{array}$} & \multicolumn{1}{l}{ Level } & Formal Approaches \\
\cline { 2 - 3 } $\begin{array}{c}\text { COMPUTING } \\
\text { AS }\end{array}$ & $\begin{array}{l}\text { Neurotransmitters, membrane } \\
\text { phenomena, action potentials } \\
\text { Biophysics of neural codes and } \\
\text { multiple codes }\end{array}$ & Biochemistry, Biophysics \\
\cline { 2 - 3 } & $\begin{array}{l}\text { Sensorial codes, decoding in } \\
\text { motor and glandular effectors, } \\
\text { coding in the Retina }\end{array}$ & $\begin{array}{l}\text { Space time System and } \\
\text { Communication Theories }\end{array}$ \\
\cline { 2 - 3 } & $\begin{array}{l}\text { Neural nets, input output } \\
\text { interaction and coordination. } \\
\text { Brain systems } \\
\text { Central neural code, cooperative } \\
\text { processes, perception of } \\
\text { universals, social-like behaviour }\end{array}$ & $\begin{array}{l}\text { Algorithmic (Logic, Symbolic) } \\
\text { Connectivistic A.I. }\end{array}$ \\
\hline
\end{tabular}

Fig. 3. Levels and formal tools in neurocybernetics.

In general, three main subdomains can be distinguished in the broad domain of artificial intelligence, as shown in Fig. 4 (MiraMira and Delgado, 2006). The actions of neurocybernetics and computational neuroscience show up in going to and from Bioinspired AI, that is the understanding of cognitive processes, from and to the more practical knowledge engineering techniques, dealing with tasks and methods. Influencing both are the more classical artificial intelligence concepts and methods, an optimistic line of thought originated in 1956, when the term artificial intelligence was coined. Some times it is called Good Old Fashion AI Representations (GOFAIR). This diagram provides for a clarifying picture of the place and role of Neurocybernetics and computational neuroscience in present day research in artificial intelligence.

In return, there are at least three paradigms of AI which are incident in the concepts and methods of Neurocybernetics. This paradigms project back to the three basic original components of neurocybernetics cited in Section 2. First, there is the Symbolic Paradigm, which is actually an updated view of Craik's proposal, corresponding to Symbolic Agents AI. Here, an internal knowledge based model of the environment allows to deal with the external reality in a more effective way. This model is to be updated with data coming from the sensory lower level representations and from the coded motor actions prior to the executions on the environment. Planning and inferences are operated by the sensory representations and by the present model of the environment, to act on said model and the coding of output actions. This is illustrated in Fig. 5.

The second AI paradigm incident in Neurocybernetics is the Intelligent Agent Paradigm. Its basic concepts can be traced back to Wiener's purposive behaviour cited in Section 2. Here, the kernel of the situated intelligence agent is a kind of finite learning automaton, holding association tables, capable of reactive and conditional coding of elementary actions. The inputs to this association computer are the results of perceptual transforms of the sensory data.

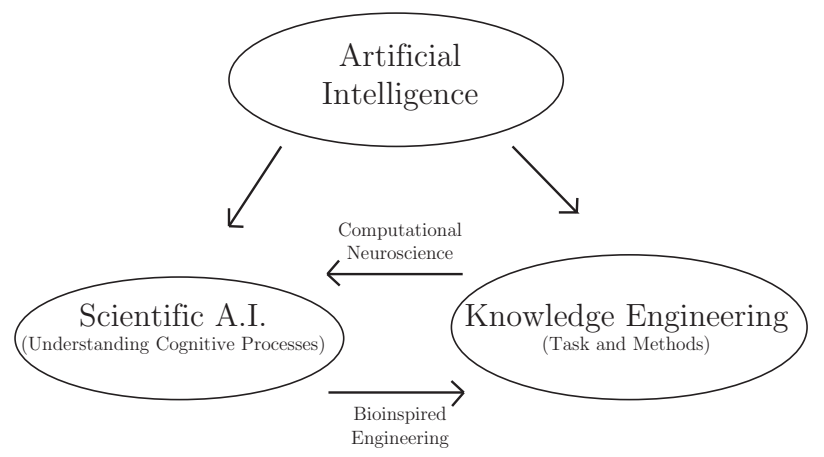

Fig. 4. Subdomains of the domain artificial intelligence and the place of neurocybernetics as a link. 


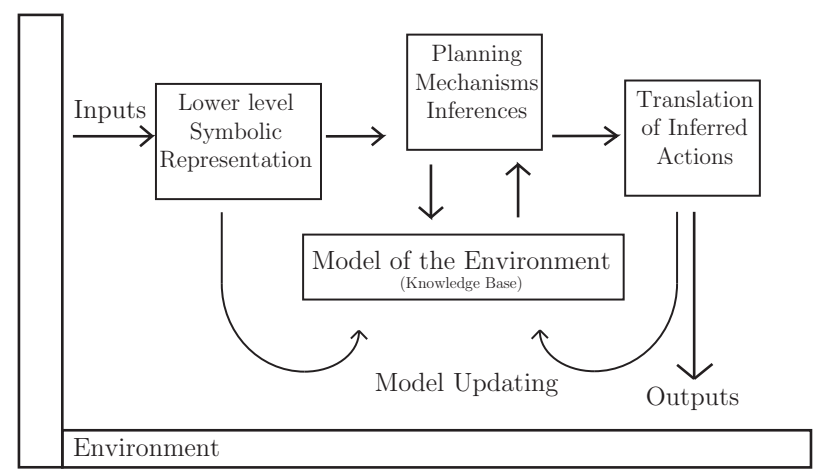

Fig. 5. Updated view of Craik's proposal. (Symbolic Agent A.I.)

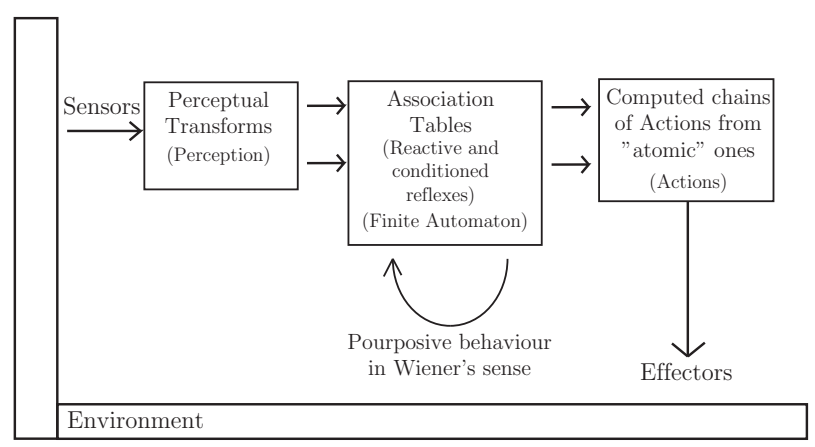

Fig. 6. Architecture of a situated intelligent agent system.

The output feeds the computation of chains of actions to go to the effectors. Notice that the main feedback loop controlling the system is an external one, determined by the situation of the agent in the environment. This type of architecture is illustrated in Fig. 6.

Finally, there is the Connectivistic Paradigm, which sprung from the original McCulloch and Pitts paper and the later Perceptrons and Artificial Neural Nets. Here, though we are far from the original meaning given to formal neurons in 1943 and to posterior work of McCulloch and collaborators, it is typically accepted that networks of artificial neurons (many of them, variants of Perceptrons) are capable of solving classes of artificial intelligence problems in a distributed, granular way. This claim is actually based on the two basic and very important translating operations performed by the human operator, external to the net: an abstraction of the observed data to generate numerical labelled variables (or input lines) and a retranslation of numerical solutions on output labelled lines or variables into a subset of the natural language, to provide classes in which the

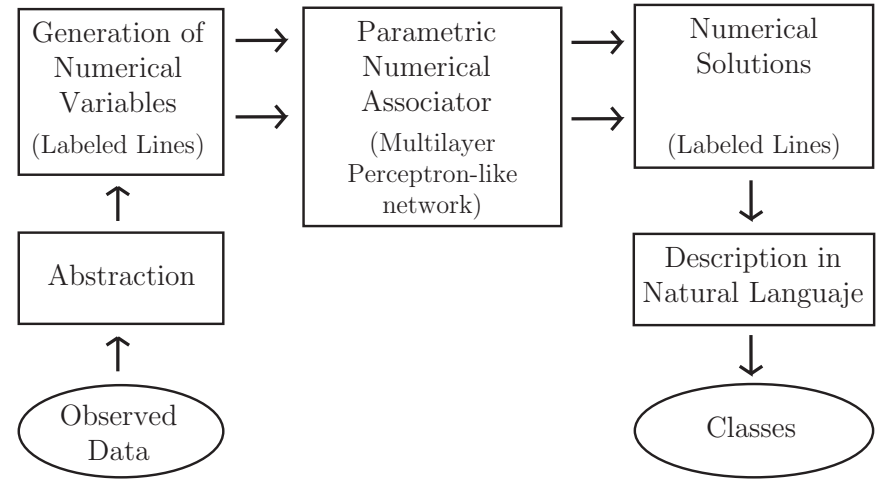

Fig. 7. Typical connectivistic A.I. application architecture of an artificial neural net.

original observed data fit. In between, an ANN (Artificial Neural Network) is actually a parametric numerical associator which can learn (modify weights), having the nature of a multilayer Perceptron. The typical connectivistic artificial intelligence architecture is shown in Fig. 7.

As it can be immediately concluded, artificial neural nets as they are nowadays understood, do not provide for theories or models of the nervous systems, but rather, they are a pure computing tool, that should always be compared with other for the solution of specific problems.

As a synthesis of the aims of neurocybernetics and of bioinspired AI, we shall refer to a diagram which reflects the aims of bringing together neuroscience and AI, in an effort to clarify and to increase our understanding of the nervous system. And also, to develop better and more sophisticated computing tools in our benefits. The diagram is shown in Fig. 8. On the left, there are the different levels of description corresponding to the structures and components. On the right, a similar representation for the same levels corresponding to neural processes is shown. In both cases, arrows coming down mean reductionistic approaches, while arrows going up would reflect emergent properties. Notice that each reduction and apparent emergency requires a change in the formal language and in the interpretations by the external observer. Much care must be taken in the jumps between levels, as it was also the case for the levels and formal tools in Neurocybernetics (Fig. 3). Trying to describe and explain cognitive processes in terms of neurons and neural nets languages is too irrealistic, a jump similar to trying to describe the Theory of Computation or Data Structures and Algorithms in terms of hardware.

COMPUTING COGNITIVE A.I.

NEUROCYBERNETICS

\begin{tabular}{|c|c|c|}
\hline $\begin{array}{c}\text { Theory of Computation Representation } \\
\text { of Goals, Believes, Purposes } \\
\text { Principle of rationality (Leibniz) }\end{array}$ & $\begin{array}{c}\text { Mind and Feelings } \\
\text { Natural Language } \\
\text { Coherent Description of Cognitive Processes }\end{array}$ & $\begin{array}{c}\text { Knowledge } \\
\text { level }\end{array}$ \\
\hline$\downarrow \uparrow$ & $\downarrow$ & \\
\hline $\begin{array}{l}\text { Data Structures Algorithms } \\
\text { Formal Languajes } \\
\text { Command and Control }\end{array}$ & $\begin{array}{l}\text { Perception Codes in Cortex } \\
\text { Role of brain regions } \\
\text { (i.e. cerebellum) } \\
\text { Reticular Formation }\end{array}$ & $\begin{array}{c}\text { Symbolic } \\
\text { level }\end{array}$ \\
\hline$\downarrow \quad \uparrow$ & $\downarrow$ & \\
\hline $\begin{array}{l}\text { Hardware and Signals } \\
\text { Parallel Architectures } \\
\text { Artificial Neural Nets }\end{array}$ & $\begin{array}{c}\text { Anatomy and Physiology of Neurons } \\
\text { and Nets } \\
\text { Morphology and Function in the } \\
\text { Nervous System }\end{array}$ & $\begin{array}{l}\text { Physical } \\
\text { level }\end{array}$ \\
\hline
\end{tabular}

Fig. 8. Bridging computing science and neuroscience. 


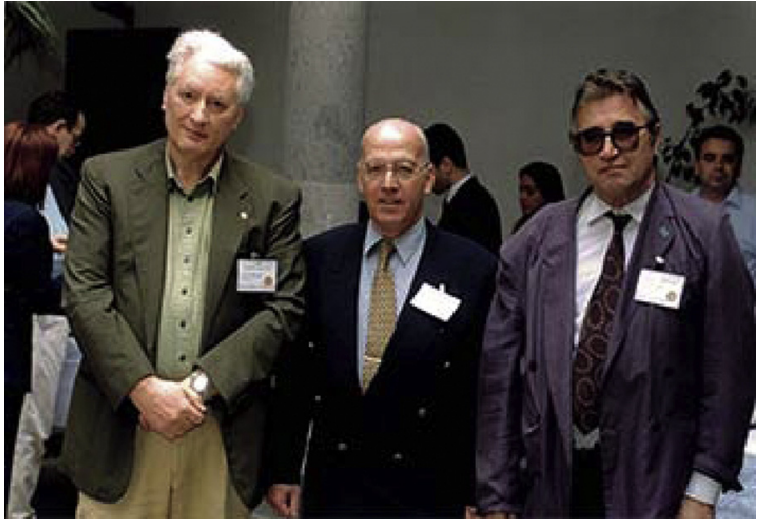

Fig. 9. From left to right, Profs. Ricciardi, Moreno-Díaz and Pichler. EUROCAST 1997. Las Palmas de G.C.

Bridging the formal tools and concepts of the two columns of the diagram at each level is an important task ahead for neuroscience and computing researchers.

\section{An acknowledgment to Prof. Luigi Ricciardi}

Prof. Ricciardi's contributions to strict Neurocybernetics, mostly to formal neural nets, belong to the beginning of his scientific career, with his master Eduardo Caianiello and other collaborators as de Luca (see, for example, (De Luca and Ricciardi, 1967, 1968; Caianiello, 1967; Caianiello et al., 1968). Later, he turned to diffusion models, and although his rich and relevant contributions to biomathematics, we will only focus on Ricciardi's relations and contributions to various computing and neurocybernetics events in Las Palmas de Gran Canaria, A Coruña and to workshops IWINAC on the interaction of natural and artificial computation (Fig. 9).

First, there are Ricciardi's contributions to Eurocast Conferences (Computer Aided Systems Theory) organized every two years by Franz Pichler and Roberto Moreno-Díaz since 1989 in Las Palmas (Spain) and Central Europe. His first contribution was to Eurocast 1993, Las Palmas, with a paper on Computational Problems for Neural Diffusion Models, coauthored by Giorno and Nobile (1994). This was the first of three years in a row that Prof. Ricciardi visit this city. The following year, 1994, he was organizing a seminar at the University of Las Palmas dedicated to the Memory of Eduardo Caianiello, who had died the year before. In 1995, he was collaborating in the International Conference "Brain Processes, Theories and Models" in the Memory of Warren McCulloch, where he joined historical cyberneticians still alive as Heinz von Foerster and Jerry Lettvin. Among the participants were Nick Leibovic, Bob Gesteland, Jose da Fonseca, Michael Arbib, José Mira, Paull Cull, Sunshuke Sato and many other well known neurocybernetics scientists.

In 1999, the University of Napoli, Linz and Las Palmas joined to organize an International Conference to commemorate 50 years of Norbert Wiener Cybernetics, a meeting chaired by Ricciardi, Pichler and Moreno-Díaz (Moreno-Díaz et al., 1999). The last disciple of Wiener, Prof. De la Riccia, then at Udine, was Ricciardi's special guest.

From 1997 on, Prof. Ricciardi contributed to all but one Eurocast Conferences, with over 20 papers by him and collaborators, chairing the Workshop on Biocomputation Methods. Contributions were mostly on Computational and Simulation Methods for Diffusion Neural Models and Neural Coding and Myosin Dynamics. He delivered twice the Opening Plenary Lecture of the Conference.

In addition, Prof. Ricciardi collaborated several times with José Mira in IWINAC meetings, giving invited lectures and with José Luis Freire, in A Coruña, where in 2008 delivered a masterly lecture that was a praeludium of his last brilliant, masterly and moving plenary lecture in EUROCAST 2011, February 9th, in Las Palmas de Gran Canaria.

This is a modest tribute to a prominent scientist, honest and serious person and friend, who enjoyed the joy of living and sharing. His legacy will remain among us.

\section{Acknowledgments}

This research has been supported by projects from MICINN (MTM2011-28983-C03-03) and CAM (P2009/ESP-1685).

\section{References}

Ashby, W.R., 1952. Design for a Brain. Wiley, Oxford, England.

Blum, M., 1961. Properties of a neuron with many inputs. In: Von Foerster, Zopf, R. (Eds.), Principles of Self Organization. Pergamon Press, New York, pp. 95-119.

Caianiello, E., 1961. Outline of a theory of thought processes and thinking machines. J. Theor. Biol. 2, 204-235.

Caianiello, E.R., de Luca, A., Ricciardi, L.M., 1967. Reverberations and control of neural networks. Kybernetik 4, 10-18.

Caianiello, E.R., de Luca, A., Ricciardi, L.M., 1968. Neural networks: reverberations, constants of motion and general behavior. In: Caianiello, E.R. (Ed.), Neural Networks. Springer Verlag, Berlin, pp. 92-99.

Cowan, J.D., 1966. Reliable Computation with Unreliable Elements. Imperial College of Science and Technology. Defense Technical Information Center, London.

Craick, K., 1943. The Nature of Explanation. Cambridge University Press, Cambridge.

De Luca, A., Ricciardi, L.M., 1967. Formalized neuron: probabilistic description and asymptotic theorems. J. Theor. Biol. 14, 206-217.

De Luca, A., Ricciardi, L.M., 1968. Probabilistic description of neurons. In: Caianiello E.R. (Ed.), Neural Networks. Springer Verlag, Berlin, pp. 100-109.

Giorno, V., Nobile, A.G., Ricciardi, L.M., 1994. On some algorithmic and computationa problems for neuronal diffusion models. In: Pichler, F., Moreno-Díaz, R. (Eds.), In: Computer aided systems theory - EUROCAST'93. Lecture Notes in Computer Science, vol. 763. Springer Verlag, Berlin, pp. 293-313.

Kilmer, W.L., McCulloch, W.S., 1964. Toward a Theory of the Reticular Formation. Electronics Research Laboratory and Air Force Cambridge Research Laboratories, U.S.

Marinaro, M., Ricciardi, L.M., Scarpetta, G., 1995. A biographical sketch of the life of Professor Eduardo R. Caianiello. Math. Japonica 41 (1), 2-5.

McCarthy, L., Minsky, L.M., Rochester, N., Shannon C.F., 1955. A proposal for the Darmouth summer research project on artificial intelligence. Tech. Report, Hannover, New Hampshire.

McCulloch, R. (Ed.), 1989. Warren S. McCulloch Collected Works. Intersystems Publications, Salinas, CA.

McCulloch, W.S., 1959. Agatha Tyche: of nervous nets - the lucky reckoners. In: Proceedings of Mechanization of Thought Processes. Her Majesty's Stationery Office, London, pp. 611-634.

McCulloch, W.S., 1974. Recollections of the many sources of cybernetics. ASC Forum $\mathrm{VI}(2), 5-16$.

McCulloch, W.S., Pitts, W.H., 1943. A logical calculus of the ideas immanent in nervous activity. Bull. Math. Biophys. 5, 115-133.

McCulloch, W.S., Papert, S.A., Blum, M., Da Fonseca, J.L., Moreno-Díaz, R., 1969. Afterdinner address: the fun of failures. Ann. N. Y. Acad. Sci. 156, 963-968.

Minsky, M.L., Papert, S., 1969. Perceptrons: an introduction to computational geometry. The M.I.T. Press, Cambridge, MA.

Mira-Mira, J., Delgado, A., 2006. A cybernetic view of artificial intelligence. Sci. Math Japonicae 64 (2), 331-349.

Moreno-Díaz, R., McCulloch, W., 1969. Circularities in Nets and the Concept of functional matrices. In: Proctor (Ed.), Biocybernetics of the Central nervous System. Little and Brown, MA, pp. 145-150.

Moreno-Díaz, R., Hernández-Guarch, F., 1983. On the biological former counterparts of logical machines. Kybern 12, 129-136.

Moreno-Díaz, R., Mira, J. (Eds.), 1996. Brain Processes, Theories and Models. The M.I.T. Press, Cambridge, MA.

Moreno-Díaz, R., Pichler, F., Ricciardi, L., Moreno-Díaz Jr., R. (Eds.), 1999. Wiener's Cybernetics: 50 Years of Evolution. Extended Abstracts.

Moreno-Díaz, R., Moreno-Díaz, A., 2007. On the legacy of W.S. McCulloch. BioSystems 88 (3), 185-190.

Pias, C. (Ed.), 2003. Cybernetics/Kybernetik. The Macy Conferences (1946-1953) vols. 1, 2. Diaphanes, Zürich /Berlin.

Piccinini, G., 2004. The first computational theory of mind and brain: a close look at McCulloch and Pitts's "logical calculus of ideas immanent in nervous activity". Synthese 141, 175-215.

Pitts, W.H., McCulloch, W.S., 1947. How we know universals; the perception of auditory and visual forms. Bull. Math. Biophys. 9, 127-147.

Ricciardi, L.M., 1994. Eduardo R. Caianiello (1922-1993). Math. Japonica 39 (1) I-XVI.

Ricciardi, L.M., Lánský, P., 2003. Diffusion models of neuron activity. In: Arbib M.A. (Ed.), Handbook of Brain Theory and Neural Networks. The M.I.T. Press, Cambridge, pp. 343-348.

Ricciardi, L.M., 2010. Uncertainty, probability and functionality in contexts of computational biology. In: Blanco, A., Castro, L.M. (Eds.), Research and Teaching 
in Computing Engineering: High Standards and Reflections. Universidade A Coruña, Coruña, Spain, pp. 82-128.

Schlatter, M., Aizawa, K., 2007. Walter Pitts and "a logical calculus". Synthese 162, 235-250.

Schnabel, C.P.J., 1965. Number of Modes of Oscillation of a Net of N neurons. Quarterly progress report N. 80. Research Laboratory of Electronics. M.I.T, pp. 253.

Rosenblueth, A., Wiener, N., Bigelow, J., 1943. Behavior, purpose and teleology. Philos. Sci. 10 (1), 18-24.
Von Foerster, H., 1974. Cybernetics of Cybernetics. University of Illinois, Urbana, IL. Von Neumann, J., 1956. Probabilistic logics and the synthesis of reliable organisms from unreliable components. In: Shannon, McCarthy (Eds.), Automata Studies. Princenton University Press, Princeton, NJ, pp. 43-98.

Wiener, N., 1948. Cybernetics. The Technology Press/John Wiley and Sons Inc., New York. 